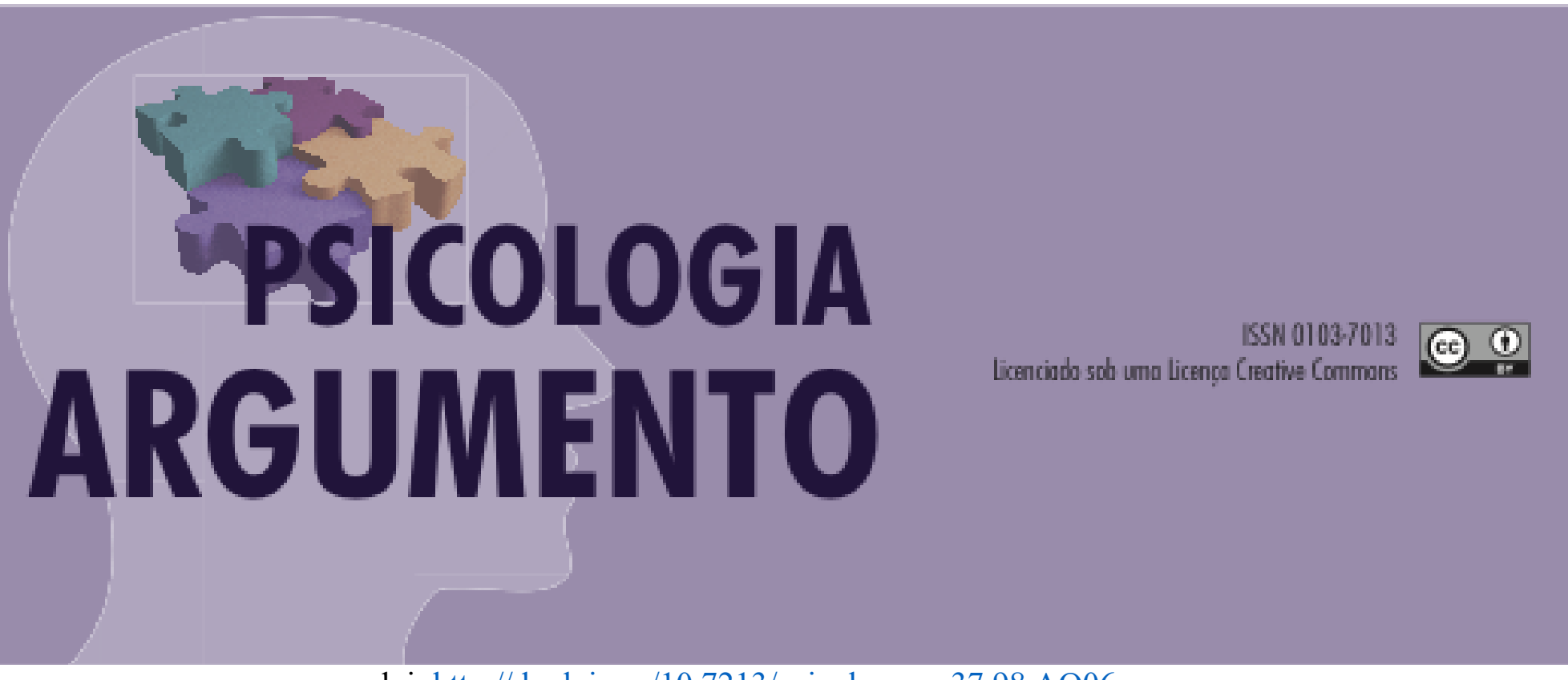

doi: http://dx.doi.org/10.7213/psicolargum.37.98.AO06

\title{
A atuação da psicologia hospitalar, breve histórico e seu processo de formação no Brasil
}

The acting in hospital psychology, a brief history and its training Process in Brazil

El rendimiento de psicología del hospital, breve historia y su proceso de formación en Brasil

Fabiane Espindola de Assis

Psicóloga, especializanda em perinatalidade e psicologia na reprodução humana. Cuiabá/Mato Grosso/Brasil. E-mail: fabiane.ea@gmail.com ORCID: http://orcid.org/0000-0003-2556-5705

Sue Ellen Ferreira Modesto Rey de Figueiredo

Psicóloga, Doutora em Oncologia, Docente da Universidade de Cuiabá (UNIC), Mato

Grosso/Brasil. E-mail: suemodesto@hotmail.com ORCID:

http://orcid.org/0000-0002-4958-5826

\begin{abstract}
Resumo
Este artigo busca apresentar um panorama a respeito da formação e da atuação do psicólogo no âmbito hospitalar, tendo em vista a sua relevância e o caráter de tratamento, considerando o indivíduo em sua totalidade. $\mathrm{O}$ artigo discorre também sobre a baixa inserção dos acadêmicos e profissionais nessa área em todo o Brasil, que tem apenas os grandes centros como referências. Essa área que poucos conhecem, apresenta multitarefas, com o objetivo de ajudar o paciente e o familiar a lidarem com os aspectos psicológicos do adoecimento e do processo de internação. A opção metodológica da pesquisa foi uma revisão bibliográfica de artigos acerca da Psicologia Hospitalar, mais conhecida mundialmente como Psicologia da Saúde. Para isso, foi feito um apanhado de diversos autores sobre o tema, e posteriormente a fundamentação entre eles com base na temática escolhida.
\end{abstract}

Palavras-chave: Psicologia; Hospitalização; Psicologia Médica; Psicologia da Saúde. 


\section{Summary}

This article seeks to present an overview regarding the formation of the psychologist's performance in the hospital context, in view of its relevance and the treatment character, considering the individual as a whole. The article also discusses the low insertion of academics and professionals in this area throughout Brazil, which has only large centers as references. This area that just known by a few people, presents multitasks, in order to help the patient and family cope with the psychological aspects of illness and hospitalization process. The methodological approach of the research was a literature review of articles about Hospital Psychology, known worldwide as Health Psychology. For this, we made an overview of various authors on the subject, and then the grounds between them based on the chosen theme.

Keywords: Psychology; Hospitalization; Psychology, Medical; Health Psychology.

\section{Resumen}

Este artículo busca presentar una visión general sobre la formación del desempeño del psicólogo en el contexto hospitalario, considerando su relevancia y el carácter del tratamiento, considerando al individuo como un todo. El artículo también analiza la baja inserción de académicos y profesionales en esta área en todo Brasil, que solo tiene grandes centros como referencias. Esta zona que pocos conocen tiene características de multitarea, con el fin de ayudar el paciente y su familia frente a los aspectos psicológicos del proceso de la enfermedad y la admisión. El enfoque metodológico de la investigación fue una revisión de la literatura de artículos sobre Psicología de la Salud. Para eso, se hizo una colección de varios autores sobre el tema, y que incluye además el razonamiento basado en el tema seleccionado.

Palabras clave: Psicología; Hospitalización; Psicología Médica; Psicología de la salud.

\section{Introdução}

O trabalho de profissionais da psicologia em instituições hospitalares públicas e privadas vem ganhando espaço diariamente, porém, ainda tem a sua identidade em construção pelas multitarefas e seu caráter multiprofissional. Contrapõem-se a medicina, com o seu modelo biopsicossocial, com o entendimento complexo acerca dos possíveis fatores predisponentes e precipitantes do adoecimento. Nessa área de atuação percebe-se que as funções desempenhadas pelos psicólogos são muito mais abrangentes do que nos modelos tradicionais de atendimento.

Segundo Mota, Martins \& Veras (2006) a psicologia da saúde tem sua contribuição não apenas com uma visão da recuperação mas sim com a inclusão da promoção da saúde, que visa a prevenção de doenças e manutenção da saúde no âmbito físico, mental, social e espiritual. No contexto hospitalar a assistência psicológica é centrada em minimizar o sofrimento provocado pela hospitalização ao paciente e seus familiares.

Em requisito acadêmico, a graduação em Psicologia geralmente é centrada no contexto de atuação clínica, restringindo a teoria e a prática aos outros campos de atuação. 
A psicologia oferece aos profissionais diversas áreas de trabalho e para que o psicólogo esteja apto para trabalhos em campos específicos, ele precisa buscar além do que a faculdade proporciona, garantindo um trabalho com maior qualidade na área desejada, sendo a área hospitalar também reflexo desse desenvolvimento.

Diante disso, este artigo visa trazer uma breve reflexão teórica a respeito do panorama da atuação do psicólogo hospitalar e a sua formação para que esta atuação ocorra de forma adequada.

\section{Breve histórico sobre o surgimento da psicologia hospitalar}

A psicologia no Império Romano tinha grande relação com a igreja católica, nessa época o conhecimento era monopolizado, e consequentemente o estudo do psiquismo também. Nesta época, Santo Agostinho e Santo Tomás de Aquino foram influenciados por obras de Platão, Aristóteles e pela Bíblia como grandes precursores do estudo psicológico, que relacionavam a alma e o corpo. É com Santo Tomás de Aquino que os estudos psicológicos rompem com a igreja católica, seguindo ao renascimento com grandes transformações no mundo das ciências e com a separação entre corpo e alma (Belotti, 2009).

Na Idade Média a igreja permaneceu em grande ligação com a psicologia. Durante séculos pessoas com deficiência mental, assim como moradores de rua eram ditos como loucos e foram afastados da sociedade e abrigados pelas igrejas, fazendo surgir assim os primeiros hospícios, que na época tinham o significado de hospitalidade ou hospedagem. Nesse local os seres que residiam eram considerados sem razão e sem direitos, com o objetivo de isola-los da sociedade. Essa parte da população passou a ser vista como preguiçosos e por isso eram submetidos ao trabalho forçado junto com prostitutas, doentes venéreos e criminosos comuns. Seguindo esse percurso, no século XVII os doentes mentais passaram a viver em hospitais e no século XVIII com Phillippe Pinel, obtiveram a substituição por manicômios, onde eram torturados, aprisionados e eram desenvolvidas experiências como formas de tratamento com esses doentes mentais. (Sanchez, Kanashiro, Evangelista \& Nunes, 2000).

Com essa configuração hospitalar na época, a medicina não tinha a sua intervenção efetiva, apenas no final desse mesmo século, com as grandes epidemias devido ao tráfico de mercadorias trazido das colônias é que a medicina ganhou força e 
ocupou o seu espaço nessas instituições. Mesma época em que, segundo Foucault, é a data do surgimento do hospital como instrumento terapêutico (Salto, 2007).

Nesse processo a psicologia começa a se estruturar e ganhar força apenas no final do século XIX, proporcionando diferentes áreas de atuação e conhecimento. A consolidação do campo da psicologia hospitalar tem ligação direta com as transformações nas instituições hospitalares que ocorreram nas primeiras décadas do século passado. Com a produção dos quimioterápicos e os instrumentos tecnológicos, o hospital assumiu uma nova postura, se configurando em um espaço de tratamento e recuperação. As enfermeiras assumem o papel antes ocupado pelas irmãs de caridade, proporcionando aos enfermos cuidados físicos, espiritual e emocional, funções estas que posteriormente foram atribuídas às assistentes sociais e aos psicólogos. (CRPSP, 2004).

Diante da dificuldade de inserção da psicologia nesse espaço hospitalar, percebese a necessidade de ressaltar mais detalhadamente essa caminhada. Apenas na década de 50 as atividades psicológicas em ambientes hospitalares se iniciaram no Brasil, porém, somente em 1962 ocorreu a regulamentação dessa profissão (Gorayeb \& Guerrelhas, 2003). Os primeiros registros nesta área datam de 1954 em São Paulo. Nesta mesma época Mathilde Neder deu início a essa atividade pioneira no Instituto de Ortopedia e Traumatologia do Hospital das Clínicas da Faculdade de Medicina da Universidade de São Paulo, com a demanda de atendimento psicológico no hospital vinda por meio da equipe médica para auxiliar crianças na adesão ao tratamento (Dittrich \& Zendron, 2001).

Esse pedido da equipe médica solicitava à psicologia uma ajuda em relação a adesão ao tratamento por parte das crianças. Assim, as primeiras atividades da psicóloga Mathilde Neder consistiam em prestar consultas psicológicas a crianças submetidas a cirurgias de coluna, bem como a seus familiares. Realizando o atendimento psicológico no pré e pós-operatório, dando assim início as atividades psicológicas desenvolvidas em hospitais no Brasil (Silva, 2006).

Esse caminhar da psicologia hospitalar se deu de forma lenta e apenas no ano de 1983, ocorreu o I Encontro de Psicólogos na Área Hospitalar, com a criação, por meio do CRP/06 (da região de São Paulo), de uma subcomissão para a discussão de temas relacionados ao campo da Psicologia Hospitalar, como suas peculiaridades, convergências e divergências em relação a outras áreas da Psicologia (Pibernat, 2006).

Diante desse processo histórico de inserção da psicologia no campo hospitalar, o Brasil tem usado o termo Psicologia Hospitalar para designar o trabalho de psicólogos 
em hospitais, porém faz-se importante ressaltar que essa denominação existe apenas no Brasil (Sebastiani, 2003; Yanamoto, Trindade \& Oliveira, 2002; Tonetto \& Gomes, 2005 apud Almeida \& Malagris, 2011). Para regularizar a concessão de título de especialistas, incluindo o título de Psicologia Hospitalar, o Conselho Federal de Psicologia (CFP) promulgou a resolução de $n^{\circ} 014$, para dar legitimidade a essa prática.

\section{Formação do psicólogo}

A graduação em psicologia proporciona uma aprendizagem "generalizada" com a formação científica-metodológica, na qual há um conjunto de desenvolvimento de técnicas para as futuras intervenções em geral voltadas para a prática clínica, sem se especializar em uma determinada área, ou seja, o curso de formação de Psicologia preza por uma formação mais generalista, o que sugere ao profissional que se forma a necessidade de se capacitar em alguma área específica por meio de cursos de pósgraduação e/ou especialização (Carvalho \& Sampaio, 1997).

Os estágios extracurriculares são uma boa alternativa para os discentes que podem conhecer melhor todas as áreas de atuação que a profissão oferece, assim ampliam o conhecimento e já despertam a atenção dos futuros profissionais para áreas especificas. Buscando estágios extracurriculares, o aluno se depara com situações que desafiam a sua prática, auxiliando no raciocínio e interesse, fato este que pode ser visto como o ponto de partida para a inserção no meio científico e de questões a serem trabalhadas (Carvalho \& Sampaio, 1997).

Essa área da psicologia é tão importante que todos os psicólogos que desejam nela atuar, devem adquirir conhecimentos específicos, através da especialização, com estudos aprofundados. Essa concepção se dá pelo fato de envolver a compreensão das teorias e métodos da psicologia da saúde e sua aplicação ao ambiente hospitalar. Um psicólogo hospitalar capacitado tende a trabalhar no campo de atuação com critérios que incluam a saúde física, o estresse, controle da dor, reabilitação de pacientes com doenças crônicas e no desenvolvimento de intervenções para problemas como cirurgia invasiva (Ogden, 2007 apud Almeida \& Malagris, 2011).

Pensando nessa formação e na importância que a psicologia hospitalar vem conquistando ao longo dos anos é que, por volta de 1968, as primeiras disciplinas relacionadas à saúde começaram a ser ministradas nos cursos de Psicologia da 
Universidade de São Paulo. A partir desse momento a Psicologia Hospitalar vem construindo a sua identidade profissional, e o seu trabalho vem sendo delineado à medida que os médicos e enfermeiros percebem que certas situações fogem ao seu conhecimento (Torezan et. al., 2013).

Assim como em outras áreas da psicologia encontramos uma multiplicidade de práticas oriundas de diferentes teorias psicológicas, que possibilitam ao profissional diferentes formas de atuação. Dessa forma a intervenção pode seguir diferentes configurações dependendo da teoria utilizada (Azevêdo \& Crepaldi, 2016).

\section{Definição e atuação dos psicólogos em hospitais}

Antes de iniciar a discussão a respeito do psicólogo hospitalar, faz-se importante ressaltar a diferença existente entre a terminologia da Psicologia da Saúde e da Psicologia Hospitalar. Na verdade, o que distingue a atuação na Psicologia da Saúde da atuação em outras áreas da Psicologia é o fato de os indivíduos atendidos por essa área terem, em geral, um problema ligado à sua saúde física, de diversas ordens ou gravidades possíveis. Já a Psicologia Hospitalar é uma área dentro da Psicologia da Saúde que tem como necessidade a intervenção precisa e adequada em um ambiente hospitalar (Gorayeb, 2010).

Segundo Almeida \& Malagris (2015) \& Salto (2010) a área da saúde na atuação da psicologia hospitalar vem crescendo devido não só ao seu caráter de tratamento, mas principalmente ao seu caráter preventivo, considerando aspectos físicos e emocionais, tendo a visão do ser humano em sua totalidade como um ser biopsicossocial. Nota-se que atualmente o governo tem-se atentado a políticas públicas de saúde mental, com promoção e prevenção, fator que têm gerado novas oportunidades em concursos públicos para psicólogos na área hospitalar, que possibilitam o empoderamento da profissão ao seu caráter de prevenção, controle e tratamento de doenças, porém ressalta a discrepância destes profissionais em hospitais públicos e privados. Muitos hospitais particulares se restringem a um ou dois profissionais, ou até mesmo nenhum, em sua grade de funcionários, podendo ser reflexo de não terem notado os benefícios e contribuições destes profissionais.

A psicologia dentro do hospital tem o objetivo de ajudar o paciente e o familiar a lidarem com os aspectos psicológicos do adoecimento e do processo de internação. Ao 
ser internado o paciente perde a sua rotina e entra num processo de limitação da sua autonomia. Dependendo do processo em que se encontra durante a internação, o paciente pode inclusive ser privado do convívio com a família por diversos fatores, o que influenciará na forma com que o paciente lidará com o processo de adoecimento e internação, tendo em vista que o apoio psicossocial é muito importante neste momento. Dentre os fatores que limitam o tempo de convívio do paciente internado com seus familiares está a internação na Unidade de Terapia Intensiva (UTI) devido ao seu horário restrito de visitação. Neste sentido, um dos papéis do psicólogo hospitalar é também o de ajudar a família a lidar com a situação e a mudança da dinâmica familiar (Sebastiani \& Maia, 2005).

De acordo com Almeida \& Malagris (2011), a UTI é sinônimo de morte para muitas pessoas, onde acontece uma luta diária pela vida. Essa percepção errônea acarreta uma forte energia negativa aos familiares dos enfermos e aos profissionais que ali trabalham na tentativa de promover um melhor atendimento ao paciente internado. Devido a essas concepções é que se faz necessário um acompanhamento psicológico para trabalhar questões relacionadas a essa impossibilidade de estar ao lado de seu ente querido. É importante também que o hospital ofereça suporte psicológico aos seus funcionários para elaborarem todas as questões envolvidas no ambiente hospitalar.

Segundo Simonetti (2004) a psicologia hospitalar tem como objetivo principal auxiliar o paciente em relação a elaboração simbólica do adoecimento, isso significa que o psicólogo valerá de seus conhecimentos técnicos para ajudar o paciente a abarcar as experiências do seu adoecimento por meio da subjetividade de cada um. É objetivo do psicólogo hospitalar minimizar as angústias e os sofrimentos, para que o paciente passe pela sua hospitalização da melhor maneira possível, garantindo o seu bem-estar. Para isso o psicólogo deve utilizar de todos os possíveis meios de comunicação com o paciente, seja através de gestos, olhares ou gemidos, sendo também porta-voz do paciente.

De acordo com a definição do Conselho Federal de Psicologia (CFP, 2010), o psicólogo Hospitalar tem nas suas atribuições a prestação de serviços de nível secundário e terciário da atenção à saúde, realizando atividades como: atendimento psicoterapêutico; grupos psicoterapêuticos; grupos de psicoprofilaxia; atendimentos em ambulatório e unidade de terapia intensiva; pronto atendimento; enfermarias em geral; psicomotricidade no contexto hospitalar; avaliação diagnóstica; psicodiagnóstico; consultoria e interconsultoria. 
Dentre essas funções exercidas pelo psicólogo hospitalar, há a necessidade do acompanhamento de casos de transtornos mentais e de sintomas que apareçam durante a hospitalização (como estado depressivo e ansiedade acentuada), geralmente como uma interconsulta advinda da equipe médica. Também é solicitado ao psicólogo, em casos específicos, a realização do psicodiagnóstico verificando a necessidade de informar a família a respeito de um acompanhamento psicológico posterior a alta hospitalar (Simonetti, 2004).

Com essas informações e corroborado pelo Conselho Federal de Psicologia (CFP) por meio da Resolução 13/07, percebe-se que o psicólogo desenvolve multitarefas, tendo como principal atividade a avaliação e o acompanhamento de mudanças psíquicas dos pacientes em internação e que serão submetidos às cirurgias. Ele também proporciona a promoção e/ou recuperação da saúde mental por meio de intervenções nas relações do paciente consigo, com o médico, com seus familiares, com o processo de adoecer e a hospitalização. Tendo em vista todos os aspectos físicos e emocionais que a situação pode gerar. Este profissional também pode atuar em instituições de ensino superior e em centros de estudos de pesquisa, visando o aperfeiçoamento ou a especialização do seu trabalho e de outros profissionais nessa área.

Conforme Almeida e Malagris (2011) nas instituições hospitalares o psicólogo não realiza os seus atendimentos como no modelo tradicional clínico o de setting terapêutico. É o psicólogo que se desloca até o paciente, o espaço físico não é privado podendo ser interrompido a qualquer momento por médicos e enfermeiros cumprindo suas funções que são muito dependentes de horários rigorosos para suas intervenções (como a aplicação da medicação no horário pré-determinado), o que pode acarretar a interferência do atendimento psicológico se este estiver ocorrendo exatamente no horário prescrito da medicação. O psicólogo deve ter ciência que sofrerá interrupções, adiantamentos e cancelamentos que estarão fora do seu controle, pois a prioridade de tratamento médico e medicamentoso deve ser respeitada em prol da saúde física do paciente.

No hospital, é importante que o psicólogo trabalhe com uma equipe multidisciplinar, esta equipe estará atenta a todas as questões do paciente, inclusive da necessidade de um acompanhamento psicológico. O contato constante com este paciente possibilita perceber situações e comportamentos que estejam fora do padrão, assim o psicólogo por meio de uma interconsulta fará o atendimento solicitado. Também é 
importante a realização de encontros entre a equipe para a discussão dos casos atendidos, bem como a atuação do psicólogo de forma direta com a equipe, aliviando o estresse psíquico dos médicos, enfermeiros e todos os profissionais envolvidos. Nessa atuação com a equipe hospitalar o psicólogo possibilita melhores atendimentos, tendo em vista que os profissionais dessa área trabalham muito com a dor e a finitude, podendo se sobrecarregar com muitos sentimentos, dentre eles os mais presentes são: ressentimento, angústia e frustrações (Pereira, 2003).

Essa sobrecarga de trabalho perante a equipe de saúde se dá pelo fato de existir o cuidado com o paciente na tentativa de propiciar uma boa qualidade de vida, relacionado a um tratamento de alívio e controle dos sintomas. Isso gera conflitos que coexistem dentro da instituição hospitalar como lidar com a vida e a morte, com o bem-estar e o ataque à doença e a questão da cura e do cuidar (Kóvacs, 2010). Por isso o psicólogo auxilia na vivência com os conflitos internos, na elaboração das perdas, na fragilidade do ser e no estresse constante, trabalhando com a identificação e o alívio desses sujeitos, favorecendo uma melhor qualidade de vida para eles. Para Tonettto \& Gomes (2007) o psicólogo também auxilia no vínculo mãe e bebê, prepara para cirurgia, trabalha aspectos da sexualidade envolvidos na doença e no tratamento, assim como também orienta os pais sobre como informar seus filhos a respeito da hospitalização ou da morte.

Ainda de acordo com Kóvacs (2010), ela se refere também ao fato destes profissionais que lidam diariamente com a morte, passarem a ter dificuldades com a vivência diária com os seus pacientes, colegas de trabalho e familiares, dificultando a resolução de problemas e acarretando sentimento de frustração, impotência e revolta. Esses sentimentos e comportamentos podem ser intensificados quando se tem vínculo com o paciente e em casos graves, como doenças incuráveis, onde a equipe prioriza a qualquer custo a vida do paciente, aumentando o estresse do profissional e os sentimentos extremamente dolorosos.

Pereira (2003) prossegue se referindo que o psicólogo diante do médico deve saber se posicionar e sustentar os seus posicionamentos, de forma clara, objetiva e coerente. Evitando barreiras para as suas futuras intervenções e conscientizando os demais profissionais da importância do seu trabalho nesta área. 


\section{Considerações finais}

$\mathrm{Na}$ revisão bibliográfica realizada, pôde-se observar o lento caminhar das instituições hospitalares nas propostas de mudanças atinentes à visão dos processos relacionados à saúde, ao adoecimento e a necessidade de inserção do psicólogo hospitalar. Faz-se necessário conhecer o status dessa profissão no Brasil para que se possa compreender o seu desenvolvimento.

Considerando as variáveis apresentadas a partir da formação das instituições hospitalares e o processo de inserção do profissional psicólogo, podemos observar o predomínio do modelo clínico nas instituições de ensino de acordo com as grades curriculares, porém atualmente em processo de mudança para um modelo de atenção integral à saúde/biopsicossocial, reflexo esse da inserção da disciplina de psicologia em outros cursos da área da saúde na graduação, fator que evidencia a importância da atuação e possibilita aos futuros profissionais um trabalho multidisciplinar com maior qualidade.

O psicólogo no hospital vem conquistando o seu espaço diariamente, mostrando a sua importância para os demais profissionais, e sempre respeitando a integridade física do paciente, assim como busca compreender o papel das variáveis psicológicas sobre a manutenção da saúde, o desenvolvimento de doenças e comportamentos associados às doenças, dando assistência para o paciente, a família e a equipe. Resultando em um trabalho de extrema importância para a promoção da saúde, prevenção e tratamento de doenças.

Sabe-se que são necessários avanços, principalmente na inserção destes profissionais nas unidades hospitalares do Brasil, pois mesmo que exista um projeto de lei que defenda a presença do psicólogo hospitalar, nem todas as unidades contam com esse serviço em sua rede de atendimento. Sendo assim, percebe-se também que ainda há muito espaço para o estudo aprofundado e para a ampliação da formação dos fundamentos dessa área.

\section{Referências}

Almeida, R. A. \& Malagris L. E. N. (2011). A prática da psicologia da saúde. Revista da Sociedade Brasileira de Psicologia Hospitalar, 14(2), 183-202. 
Almeida R. A. \& Malagris L. E. N. (2015). Psicólogo da Saúde no Hospital Geral: um Estudo sobre a Atividade e a Formação do Psicólogo Hospitalar no Brasil. Psicol. cienc. prof. [on-line]. 2015, 35 (3), 754-767, 2015. Disponível em: $<$ http://www.scielo.br/scielo.php?script=sci_arttext\&pid=S141498932015000300754\&lng=en\&nrm=iso $>$. (Acessado em 14/12/2016).

Azevedo A. V. S. \& Crepaldi M. A. (2016). A Psicologia no hospital geral: aspectos históricos, conceituais e práticos. Estud. psicol. [on-line]. 2016, 33 (4), 573-585, Disponível em: $\quad<$ http://www.scielo.br/scielo.php?script=sci arttext\&pid=S0103166X2016000400573\&lng=en\&nrm=iso. . . (Acessado em 14/12/2016).

Belotti E. (2009). A evolução da ciência psicológica. Web artigos. [on-line]. 2009. Disponível em: $\quad<$ http://www.webartigos.com/artigos/a-evolucao-da-cienciapsicologica/27551/>. (Acessado em 03/2016).

Carvalho M. T. M. \& Sampaio J. R. (1997). A formação do psicólogo e as áreas emergentes. Psicologia: Ciência e Profissão, 17(1), 14-19.

CFP - Conselho Federal de Psicologia. (2010). Resolução 13/07. Disponível em: $<$ http://www.pol.org.br>. (Acessado em 12/03/10).

Cytrynowicz M. M. (2016). Uma questão de saúde: trajetória da psicologia hospitalar em SP. Produção: Videcom. Documentário, 47’47'. Disponível em: $<$ http://www.crpsp.org.br/portal/comunicacao/umaQuestaoSaude/trajetoria.html>. (Acessado em 03/2016).

Dittrich A., Oliveira, L. A. \& Zendron R. C. (2011). Franco Seminério Paulo Rosas Mathilde Neder. Rio de Janeiro: Imago.

Gorayeb R. \& Guerrelhas F. (2003) Sistematização da prática psicológica em ambientes médicos. Revista Brasileira de Terapia Comportamental e Cognitiva, 5(1), 11-19.

Gorayeb R. (2010). Psicologia da Saúde no Brasil. Psicologia: Teoria e Pesquisa, 26, 115-122.

Kovács M. J. (2010) Sofrimento da equipe de saúde no contexto hospitalar: cuidando do cuidador profissional. O Mundo da Saúde, 34(4), 420-429.

Mota R. A., Martins C. G. M. \& Véras R. M. (2006) Papel dos profissionais de saúde na política de humanização hospitalar. Psicologia em Estudo, 11(2), 323-330. 
Oliveira A. C. et al. (2007). Diferentes olhares sobre psicologia hospitalar dentro de um grupo a partir do estágio acadêmico. O Portal dos Psicólogos [on-line]. 2007. Disponível em: $<$ https://www.psicologia.pt/artigos/ver_artigo_licenciatura.php?diferentes-olharessobre-psicologia-hospitalar-dentro-de-um-grupo-a-partir-do-estagioacademico\&codigo $=$ TL0103 $>$. (Acessado em 03/2016).

Pereira F. M. (2003). A inserção do psicólogo no hospital geral: A construção de uma nova especialidade. Dissertação de mestrado não-publicada, Fundação Oswaldo Cruz, Rio de Janeiro.

Sanchez F., Kanashiro M., Evangelista R. \& Nunes R. (2000). Reforma Manicomial. Disponível em: <http://www.comciencia.br/reportagens/manicom/manicom8.htm>. (Acessado em 03/2016).

Salto MCE. (2007). O psicólogo no contexto hospitalar: uma visão psicodramática. Psicologia Brasil, 5(39), 12-15.

Simonetti A. (2004). Manual de psicologia hospitalar: o mapa da doença. São Paulo: Casa do Psicólogo.

Silva L. P. P. (2006). O percurso histórico do serviço de psicologia do Hospital de Clínicas de Porto Alegre. Dissertação de Mestrado não-publicada, Curso de Pósgraduação em Psicologia do Desenvolvimento, Universidade Federal do Rio Grande do Sul. Porto Alegre.

Sebastiani R. W. \& Maia E. M. C. (2005). Contribuições da psicologia da saúdehospitalar na atenção ao paciente cirúrgico. Acta Cirurgica Brasileira, 20(1), 50-55.

Tonetto A. M. \& Gomes W. B. (2005). Prática psicológica em hospitais: demandas e intervenções. Psicologia, 36(3), 283-291.

Tonetto A. M. \& Gomes W. B. (2007). Competências e habilidades necessárias à prática psicológica hospitalar. Arquivos Brasileiros de Psicologia, 59(1), 38-50.

Torezan Z. F. et al. (2013). A graduação em Psicologia prepara para o trabalho no hospital?. Psicologia: Ciência e Profissão, 33(1), 132-145. 